\title{
RECONSTRUCCIÓN DEL PERFIL DE ÍNDICE DE REFRACCIÓN DE UNA FIBRA ÓPTICA DE SILICIO PURO POR EL MÉTODO DE LOS ELEMENTOS FINITOS Y EL PATRóN DE CAMPO CERCANO
}

\author{
Luis Fernando Castrillón Gallego*
}

\section{Resumen}

El presente trabajo muestra una técnica para reconstruir el perfil de índice de refracción (PIR) de fibras ópticas, basado en la comparación de los patrones de intensidad del modo de propagación fundamental obtenido experimentalmente y el logrado teóricamente a través del método de elementos finitos, utilizando una función de mérito para ajustar parámetros. Para validar la técnica se aplica a una fibra de núcleo de silicio puro cuyo perfil de índice de refracción ha sido medido por el método de campo cercano refractado (RNF).

\section{Palabras Clave}

Perfil de índice, fibra óptica, elementos finitos, campo cercano.

\section{Abstract}

The purpose of this work is to show a technique to reconstruct the profile of the index of refraction (PIR) of optic fiber, based on the comparision of the patterns of intensity of the mode of fundamental propagation base on experimentation, and the one obtained theoretically by means of the method of finite elements, using a merit function to adjust parameters. In order to validate

* Ing. en Instrumentación y Control del Politécnico Jaime Isaza Cadavid. Especialista en Óptica Técnica Universidad Nacional de Colombia sede Medellín. Docente ITM luiscastrillon@itm.edu.co 
the technique, a pure silicon nucleus fiber whose index of refraction has been measured by the method of the refracted field near (RFN) is applied.

\section{Key words}

Index profile, optical fiber, finite elements, near field. 


\section{INTRODUCCIÓN}

El presente trabajo sirve como base para determinar el perfil de índice de refracción de una fibra óptica de silicio puro, enmarcado en la utilización de la técnica del Campo Cercano y el método de los Elementos Finitos. El resultado del estudio muestra la correspondencia entre los datos obtenidos de la simulación teórica y las características dadas por el fabricante de la fibra óptica. Podrá observarse la posibilidad de ampliar este modelo a otras fibras ópticas, con otros perfiles de refracción que permitan en próximos desarrollos cubrir la amplia gama de perfiles de índice utilizados hoy en las fibras ópticas.

La información que se obtendrá del estudio es muy importante, dado el potencial que tiene para las comprobaciones de las características de fibra óptica adquiridas a proveedores internacionales y que al momento hacen costosas dicha verificación.

Se presenta un modelo destinado a obtener la forma del perfil de índice de una fibra óptica de silicio puro, a partir de la simulación en computador de la distribución de intensidad lumínica del modo de propagación fundamental. La ecuación de Helmholtz se resuelve mediante el método de los elementos finitos, simulando un perfil inicial de paso con parámetros típicos de los procesos de fabricación y hacen que esta ecuación se convierta en un problema matricial de valores propios generalizado. Posteriormente, se realiza la comparación entre los valores de campo eléctrico obtenidos con este perfil de índices de entrada y los valores de distribución de campo obtenidos en forma experimental con el recurso de una función de mérito, que permite ajustar iterativamente los parámetros de la simulación hasta obtener el perfil definitivo. 


\section{Preuminares}

\subsection{ANTECEDENTES}

Trabajos anteriores han desarrollado diversas técnicas para la determinación del PIR en fibras ópticas: Por mediciones de reflectividad $^{1}$, transmisión de luz incoherente ${ }^{2}$, por métodos interferométricos, mediciones del campo cercano y el método de las diferencias finitas, por medición del campo cercano y las pérdidas en curvaturas utilizando la trasformada de Wigner ${ }^{3}$, o por variaciones en los modos de intensidad para guías de onda y, finalmente, utilizando múltiples longitudes de onda para generar diversos modos de propagación.

En 1986, Katsumi Morishita ${ }^{4}$ determina el perfil de índice de refracción para una fibra mono-modo utilizando la medición del patrón de campo cercano en la propagación del modo fundamental.

N. Gisin, R. Passy y B. Perny ${ }^{5}$ caracterizan una fibra óptica mediante la medición simultánea de los campos cercanos trasmitido (TNF) y refractado (RNF). Llegando a tener una incertidumbre en la diferencia de índices $\Delta n= \pm 0,0002$ y en los parámetros geométricos de $0.1 \mu \mathrm{m}$ incluidos el núcleo y el revestimiento. La comparación se realiza a partir de los diámetros de campo modal (MDF) dada por la ecuación de Petermann II.

1 TOWSEND, P. D. Optical Effects of Ion Implantation. Revista: Rep. Prog. Phys., vol. 50, 1987, pp 501-558.

2 GLOGE, D., MARCATILI, E. A. Multimode theory of graded-core fibers. Revista: Journal Bell. Syst. Tech. vol. 52, n9 1973, p 1563 -1578.

3 DRAGOMAN, D., MEUNIER, J. P., Recovery of longitudinally variant refractive index profile from the measurement of the Wigner transform. Revista: Journal Optics Communications. vol. 153, 1998, pp 360 - 367.

4 MORISHITA, K. Index Profiling of Three-Dimensional Optical Waveguides by the Propagation-Mode Near-Field Method. Revista: Journal of Lightwave Technology. vol. LT-4, n8 August 1986, p 1120.

s GISIN, N.; PASSY, R. and PERNY B. Optical Fiber Characterization by Simultaneous Measurement of the Transmitted and Refracted Near Field. Revista: Journal of Lightwave Technology. vol. 11, n¹1 November 1993, p 1875. 
Posteriormente, Mansour y Caccavale ${ }^{6}$ reconstruyen el perfil de índice de una fibra óptica con la medición del campo cercano trasmitido. Utilizan un filtro pasa-bajos Butterworth, para remover las fluctuaciones de alta frecuencia, las cuales tienen severos efectos en el cálculo del perfil de índice a partir de la medición de la intensidad de campo. Para el caso de una fibra mono-modo obtienen un error del $23 \%$.

P. Torres y A. Guzmán ${ }^{7}$ utilizaron el método de los elementos finitos en un amplificador de fibra óptica dopada con erbio para analizar los cambios de IR en una sección transversal.

Von Bibra y Ann Roberts ${ }^{8}$ en 1997 presentan la distribución del índice refractivo para una fibra mono-modo de perfil gradual a partir de sus modos de intensidad.

F. Caccavale ${ }^{9}$ presenta en el año 1998, la reconstrucción del perfil de índice de una guía óptica plana a partir de la medición del campo cercano y el método de las diferencias finitas. Utiliza un algoritmo para resolver la ecuación de onda de Helmholtz escalar y, mediante una función de mérito determina el perfil de índice, comparando las distribuciones del campo eléctrico.

Ruth Meneses ${ }^{10}$, en su trabajo de grado del año 2000 presenta los montajes y experiencias prácticas para, con varias fibras ópticas,

6 MANSOUR, I., CACCAVALE, F., An Improved Procedure to Calculate the Refractive Index Profile from the Measured Near-Field Intensity. Revista: Journal of Lightwave Technology. vol. 14, n³ March 1996, $\mathrm{p} 432$.

7 TORRES, Pedro, GUZMAN, A., Amplificador de fibra óptica dopada con Erbio. Memorias Cuarto Encuentro Nacional de Óptica Universidad Nacional de Colombia Junio 29-Julio 1 de 1994.

8 BIBRA, Mark L. Von and ROBERTS, Ann, Refractive Index Reconstruction of Graded Buried Channel Waveguides from their Mode Intensities, Revista: Journal of Lightwave Technology. vol. 15, $\mathrm{n}^{\circ} 9$ September 1997, pp 1695-1699.

9 CACCAVALE, F., A finite differences method for the reconstruction of refractive index profile from near-field measurements. Revista: Journal of Lightwave Technology. vol. 16, n ${ }^{\circ}$ July 1998 , pp $1348-1352$

10 MENESES, Ruth del Socorro, Verificación del perfil de índice de refracción de una fibra óptica monomodo por el método de elementos finitos y la técnica de campo cercano, VII Encuentro de Optica Universidad del Quindío Armenia. Armenia 2004: Ediciones electrónicas de la Academia Colombiana de Ciencias Exactas Físicas y Naturales, 2000. 
excitar y capturar el modo fundamental mediante la técnica del campo cercano. El trabajo de Meneses tiene entre otras virtudes, haber presentado las condiciones necesarias para que el modo fundamental se presente: fibra mono-modo y fuente de luz láser de $630 \mathrm{~nm}$ y, por ello, esta investigación es continuación de aquel. El presente trabajo participó del VIII Encuentro Nacional de Óptica realizado en Popayán 2002.

\subsection{JUSTIFICAGIÓN}

Las características más importantes de las fibras ópticas, tales como ancho de banda, tamaño del spot, condición de mono-modo, coeficiente de acoplamiento entre guías de onda, todas ellas características de propagación, pueden ser predichas por su perfil de índice refractivo (PIR). Tanto los fabricantes, quienes deben tener especial control sobre los procesos de fabricación de dichas fibras ópticas, como quienes las adquieren para los montajes e instalaciones en comunicaciones, están interesados en conocer con eficiencia y precisión el PIR. Sin embargo, la medición de este perfil suele ser generalmente $\operatorname{costoso}^{11} \mathrm{y}$, además, el orden de magnitud de diferencia entre el índice de refracción del núcleo y el revestimiento se encuentra alrededor de $10^{-4}$, haciendo las técnicas de medición exigentes en precisión.

La técnica del campo cercano refractado es la recomendada en la medición del índice de refracción, según la norma UIT G.650 (03/93), es referida por el TIA (Asociación de Industrias de las Telecomunicaciones, por sus siglas en inglés) y, además, por ser de bajo costo su implementación en relación con la del campo cercano trasmitido (TNF)

El desarrollo de las técnicas algorítmicas permite realizar grandes cálculos y hoy son apropiadas como herramientas de gran

11 GISIN, N,, PASSY R. Perney B-. A., Optical Characterization by Simultaneous Measurement of the Transmitted and Refracted Near Field, J. Lightwave Technology, vol 11, $\mathrm{n}^{\circ} 711$ Nov. 1993. 
utilidad para determinar el índice de refracción de una fibra óptica. En general, muchos de los trabajos anteriores referidos hacen uso de la simulación, filtraje, digitalización de imágenes y demás cálcullos que serían arduos, complejos y cíclicos sin la herramienta del computador.

Este trabajo comprende la elaboración de un software para la determinación del perfil de índice de una fibra óptica, validándose el método sobre una fibra mono-modo con las siguientes características:

Fibra con perfil de paso, de silicio puro

Radio del núcleo: $4.6 \mu \mathrm{m}$,

Índice del núcleo nco $=1,4571$

Índice del revestimiento, $\mathrm{ncl}=1.4511$,

Longitud de onda utilizada para la simulación, $\lambda=1300 \mathrm{~nm}$

Se espera sea desarrollada más profundamente la técnica de reconstrucción para que ésta se aplique y amplíe para el caso de fibras ópticas especiales y de guías de onda dieléctricas.

El documento final incluye:

- El software implementado para la fibra antes descrita

- Los resultados obtenidos mediante la utilización de la técnica del campo cercano y el método de los elementos finitos

\subsection{Metodología}

Fundamentalmente, el proceso desarrollado es la comparación de dos distribuciones de intensidad de campo: la primera, experimental, obtenida mediante la medición del campo cercano refractado del modo-fundamental en una fibra óptica con las especificaciones anteriores, esta imagen es llevada a un computador mediante una cámara CCD y posteriormente digitalizada. A partir de esta imagen, se levanta la distribución gaussiana de 
luminosidad, que luego se registra en un archivo de datos de dos columnas: intensidad y posición radial.

La segunda distribución se obtiene de una simulación del modo fundamental sobre una fibra óptica virtual con las mismas características anteriores. Para esta simulación se ingresan los valores de radio, longitud de onda, tipo de perfil de índice; la distribución de campo simulada de la sección transversal es discretizada con elementos triangulares del método de elementos finitos. Posteriormente, se soluciona la ecuación de onda en el modo fundamental, la cual se ha convertido en una ecuación de valores propios (este desarrollo algorítmico fue cedido por el investigador Ph. D. Pedro I. Torres Trujillo de la Escuela de Física de la Universidad Nacional de Colombia, Sede Medellín). Se obtiene entonces, otra distribución de campo, la simulada, que es llevada a un archivo de datos, con la intensidad y su posición radial.

Para validar la técnica utilizada, se comparan las distribuciones de intensidad de campo, tanto la experimental como la teórica mediante la función de mérito. Los valores de inicio, en la simulación, asignados a los índices de refracción máximo y mínimo son reasignados por el software cuando la desviación entre los perfiles es grande; con esta variación, se construye un nuevo perfil y nuevamente se soluciona la ecuación de Helmholtz hasta lograr que el error en la función de mérito sea mínimo. Cuando esto ocurre, las características de la fibra óptica: radio del núcleo, índices de refracción, longitud de onda se hacen más próximos a los datos del perfil experimental.

Esta técnica de reconstrucción del perfil es versátil, pues permite construir cualquier tipo de perfil de índice: paso, gradual $\mathrm{u}$ otras formas.

La figura 1 muestra el desarrollo descrito en forma de diagrama de flujo para la reconstrucción del perfil. 
Figura 1. Diagrama de flujo de RECONSTRUCCIÓN DEL PERFIL dE INDICE

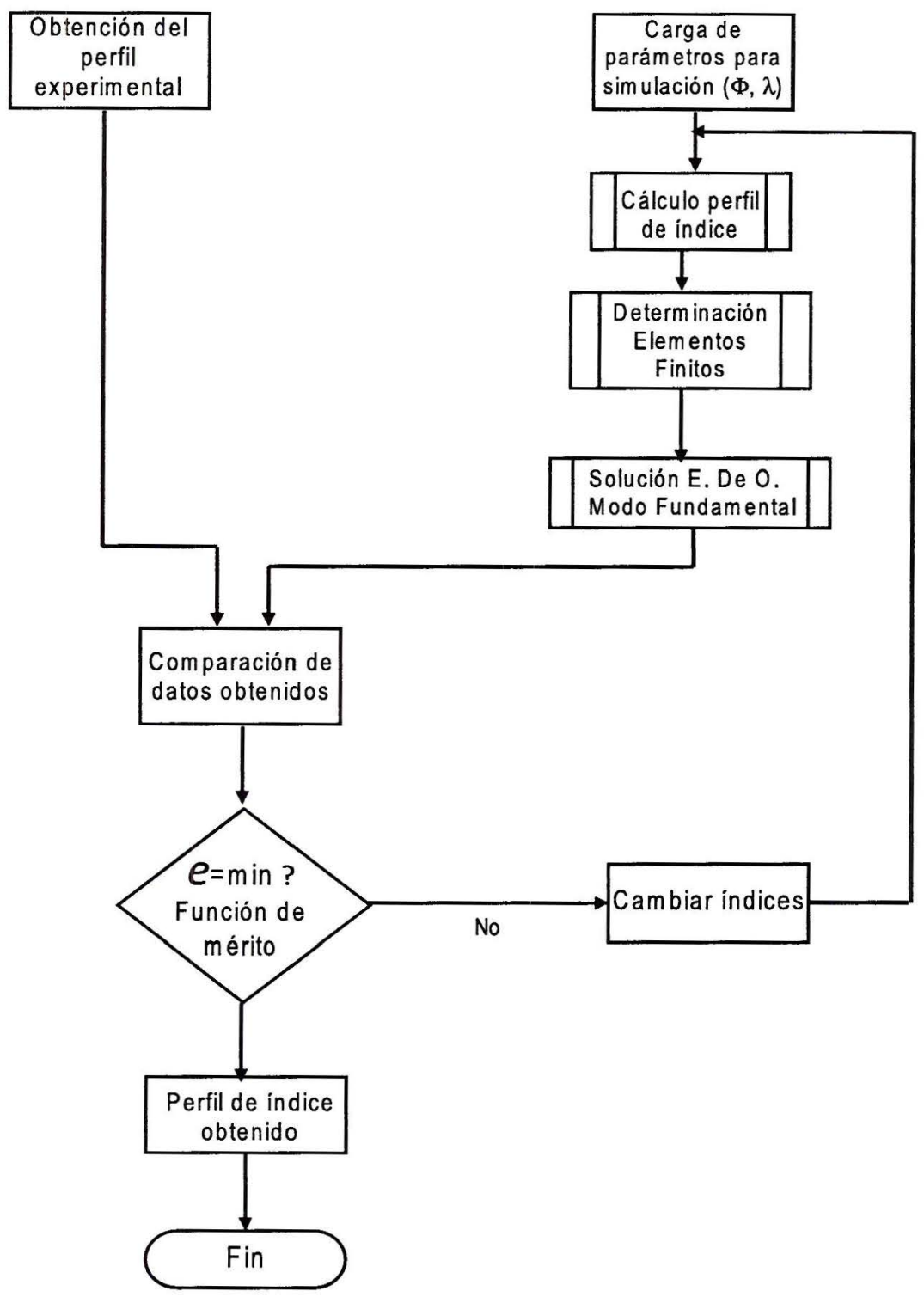




\section{Determinación del Perfll de indice de refracción}

\subsection{Perfil de indice experimental}

Para la medición del campo cercano refractado se utilizó un montaje experimental que permite capturar la distribución de intensidad de la luz de manera directa y cercana del campo trasmitido en el modo fundamental y, posteriormente, digitalizada mediante software.

Una fuente de luz láser es focalizada al centro de la fibra óptica mediante un objetivo. La fibra utilizada es una fibra mono-modo, por lo tanto, la única distribución de intensidad a la salida de la misma será el modo fundamental. Esta distribución es luego capturada por la cámara CCD, no sin antes haber sido dirigida a un sistema óptico de amplificación y polarización.

La Figura 2 muestra la disposición de un montaje para la captura del modo fundamental, mediante la técnica del campo cercano.

Figura 2 MontaJe eXPerimental para el CAMPo Cercano trasmitido

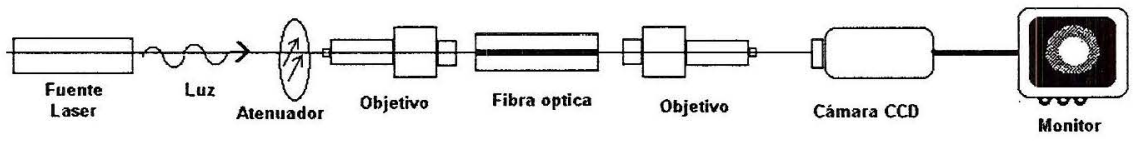

Posteriormente, la imagen es digitalizada y llevada a un archivo de datos los valores de distribución de intensidad de dicha imagen.

\subsection{Perfil de índice simulado}

Para la generación del perfil de índice refractado (PIR) de manera simulada, se utilizaron algunas rutinas previamente 
diseñadas. En ellas, se introducen los valores: Radio del núcleo (Rco), Radio del revestimiento (Rcl), Índice de refracción del núcleo (nco), Índice de refracción del revestimiento (ncl) y la longitud de onda de trabajo ( $\mathrm{\lambda}$ ). Se generan áreas triangulares (elementos finitos), de la sección trasversal, cada una de estas áreas tendrá una asignación de coordenadas radiales y un índice de refracción, este último según la ecuación de perfil de índice de una fibra óptica que viene dada por la ley de perfil de potencia

$$
n(r)=\left\{\begin{array}{c}
n_{c o} \sqrt{1-2 \Delta\left(\frac{r}{a}\right)^{\alpha}} \\
n_{c o} \sqrt{1-2 \Delta}
\end{array}\right.
$$

Con:

$n(r)$ : Perfil en función del radio

nco: Valor del Índice del núcleo

$n c l$ : Valor del Índice del revestimiento

$\Delta=\underline{n c o-n c l}$

nco

$r$ : Centro de cada elemento triangular

$a:$ Radio del núcleo

a: Exponente para determinar la forma del perfil

Si $\alpha=0$ el perfil de la fibra será de paso, si $\alpha=1$ el perfil será triangular; para valores superiores, el perfil tendrá una variación gradual.

Se genera luego el modo fundamental sobre la fibra simulada, arrojando los vectores de campo eléctrico y las coordenadas de dichos vectores. Se almacena en un archivo la intensidad de campo eléctrico junto con su par: posición radial.

La Figura 3 muestra el diagrama de flujo de la generación simulada del perfil de índice de refracción. 
Figura 3. Diagrama de flujo para la simulación del modo fundamental y PIR

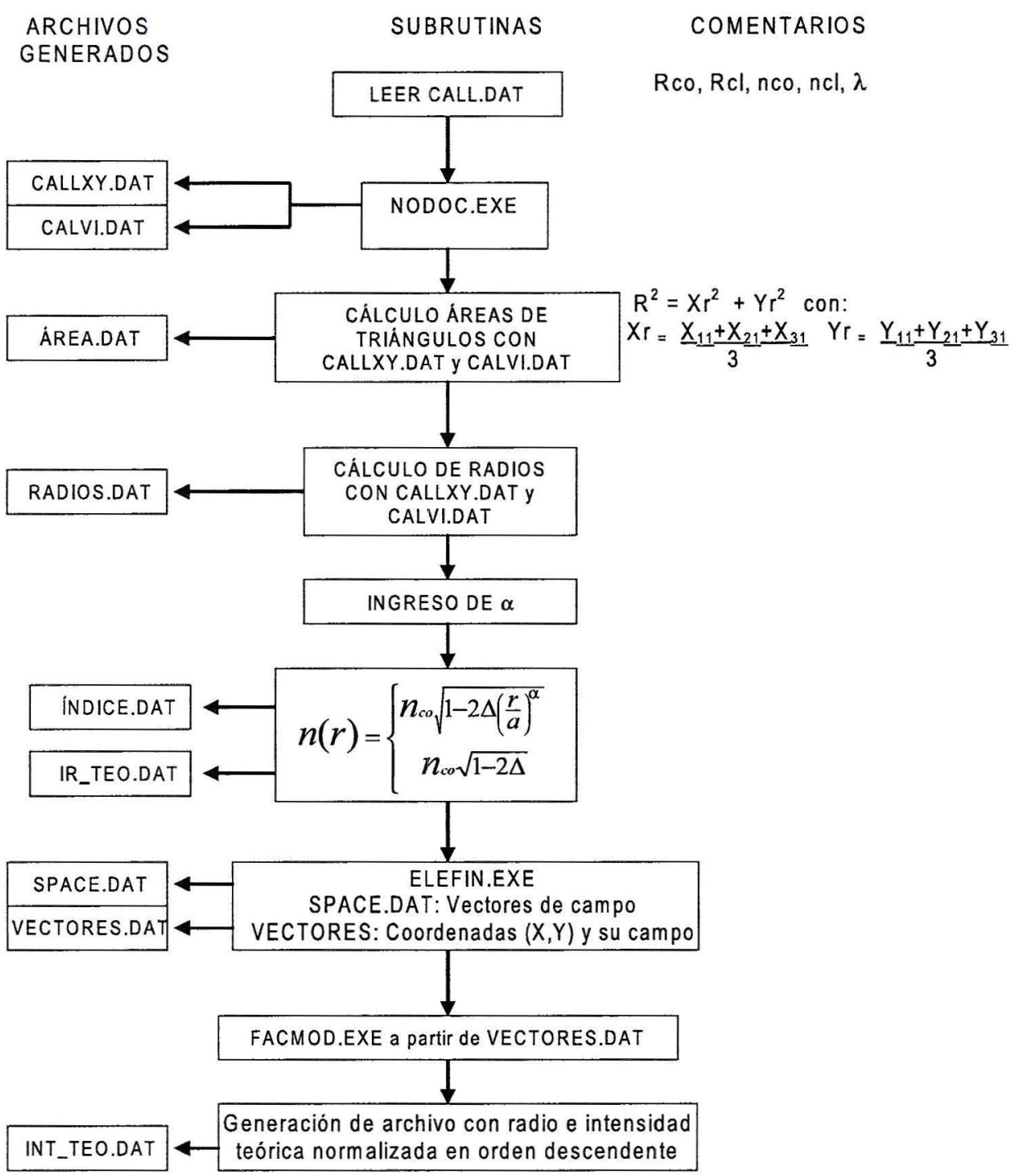




\subsection{Método de elementos finitos}

La ecuación que describe la propagación de la luz y cuando el medio es una fibra óptica con índice de refracción $n(x, y)$, es la ecuación de onda de Helmholtz escalar,

$$
\left(\nabla_{t}^{2}+k_{o}^{2} n(x, y)-\beta^{2}\right) \psi=0
$$

La función $\psi$ será, la función de distribución de campo eléctrico $E$, y viene dada por :

además,

$$
E=\sqrt{I}
$$

$$
\nabla_{t}^{2}=\frac{\partial^{2} E}{\partial x^{2}}+\frac{\partial^{2} E}{\partial y^{2}}
$$

El método de Elementos Finitos (FEM, por sus siglas en inglés), corresponde a la discretización del medio continuo y de dimensión finita. Para el caso de la fibra óptica, la sección trasversal se asume lineal e isotrópica; este continuo se divide en un número finito de partes (elementos finitos), cuyo comportamiento se especifica mediante un número finito de parámetros y la solución del sistema completo (ensamblado de los elementos) cumplirá las mismas reglas que se aplican a los problemas de tipo discreto. Los elementos están conectados entre sí mediante un número discreto de puntos llamados nodos y situados en sus contornos. Se toma un conjunto de funciones que definan de manera única cada elemento finito y que sea función de los puntos nodales.

Dada la simetría radial de la fibra óptica y la característica bidimensional de su sección transversal, los elementos suelen tomarse triangulares, aunque, podrían, en principio tener otra forma. Los nodos (vértices de los triángulos) se numeran siguiendo algún criterio. Cuando el número de vértices va creciendo, el polígono generado por el contorno de los vértices se aproxima más y más al contorno real. 
Se debe garantizar en la escogencia, la continuidad del potencial o de la función de onda, imponiendo la continuidad en los vértices de los triángulos. Las mallas triangulares pueden ser no uniformes, de modo que ciertas regiones, allí donde se esperan variaciones más bruscas del campo, estén recubiertas más densamente de elementos, como es el caso del núcleo de la fibra.

La Figura 4 muestra la ubicación del método de elementos finitos, en el algoritmo de reconstrucción del perfil de índice.

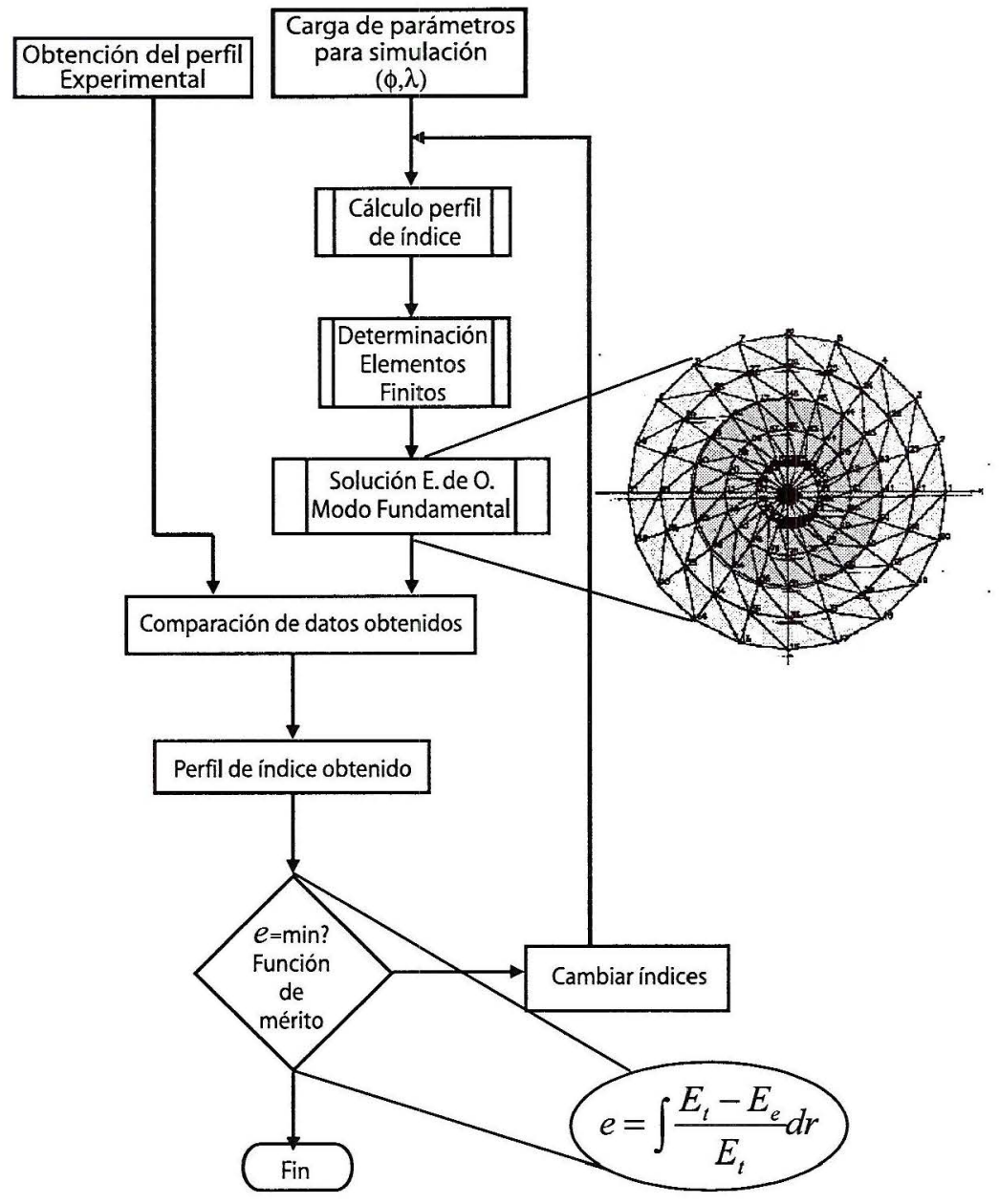


Para la realización de la discretización triangular debe imponerse como condición la continuidad del campo (eléctrica y magnética) en los nodos. Cada zona triangular se entender homogénea y representada por el baricentro de cada triángulo. $\mathrm{Al}$ aplicar el cálculo variacional a la ecuación (2) se obtienen las condiciones de frontera para los modos TE y TM contribuyentes de los modos LP (generados en fibras ópticas por su simetría), obteniéndose una integral de superficie y de línea para cada modo. La funcional $\Gamma\left(\Gamma=\Sigma_{e} \Gamma\right)$ obtenida será la suma de las contribuciones de regiones homogéneas, resultantes de la interpolación del área transversal en elementos triangulares homogéneos.

La funcional $\Gamma$ debe ser minimizada para cada elemento y se obtienen los valores de campo (TM ó TE) para los tres nodos de cada elemento finito triangular obteniéndose según sea el caso (TM ó $\mathrm{TE}$ ) el número de onda $\mathrm{K}_{\mathrm{o}}$ o la constante de propagación $B^{12}$.

\subsection{Función de MERITO}

El error relativo $e$ entre la distribución de campo eléctrico excitado el modo fundamental y obtenida en forma experimental mediante la técnica del campo cercano, y la distribución de campo eléctrico del modo fundamental de la fibra simulada, es obtenida mediante la ecuación (5), llamada función de mérito:

$$
e=\int \frac{E_{t}-E_{e}}{E_{t}} d r
$$

Con:

e: $\quad$ Error (función de mérito)

$\mathrm{E}_{\mathrm{t}}(\mathrm{n})$ : Campo eléctrico teórico normalizado (simulado)

$\mathrm{E}_{\mathrm{e}}$ : $\quad$ Campo eléctrico experimental normalizado

12 TORRES, P., GUZMAN, A., Complex finite element method applied to the análysis of optical waveguide amplifiers. Revista: Journal of Lightwave Technology. vol. 15, $\mathrm{n}^{\circ} 31997, \mathrm{p} 546$ 
La función de mérito compara los valores de campo normalizados y discretizados y ello permite la comparación algorítmica analítica para ir determinando el error.

La Figura 4 muestra la función de mérito en el algoritmo de reconstrucción del perfil de índice.

\section{Resultados y discusión}

Las Figura 5 y Figura 6 muestran los resultados obtenidos (experimental ..... y simulada -), para datos iniciales de: Rco $=5,2 \mu \mathrm{m}$ y $\lambda=1300 \mathrm{~nm}$.

FIGURA 5. PERFIL DE INTENSIDADES PARA RCO $=5,2 \mu \mathrm{m}$ y $\lambda=1300 \mathrm{~nm}$ EN SIMULACIÓN

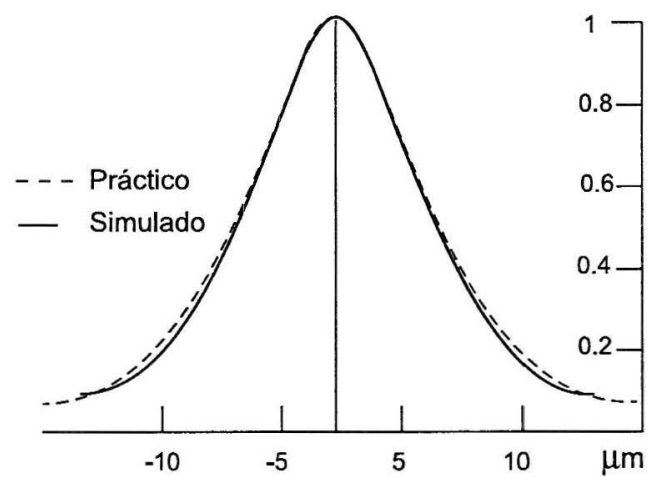

FIGURA 6. PERFIL DE IR PARA Rco $=5,2 \mu \mathrm{m}$ y $\lambda=1300 \mathrm{~nm}$ EN SIMULACIÓN

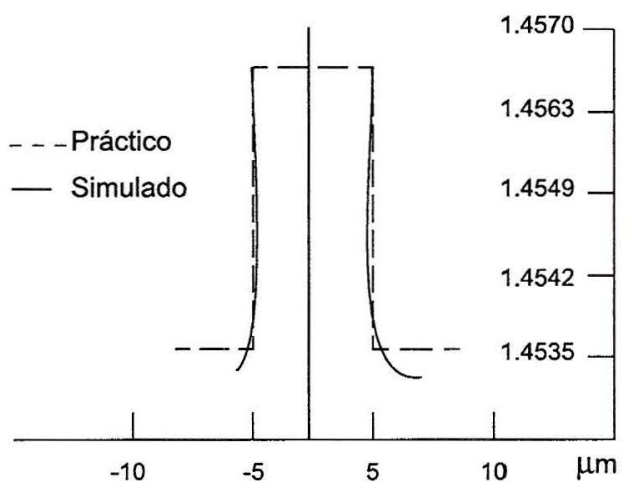


Las Figura 7 y Figura 8 muestran los resultados obtenidos (experimental -..-- y simulada - $\longrightarrow$ ), luego de varias simulaciones: Rco $=4,8 \mu \mathrm{m}$ y $\lambda=1300 \mathrm{~nm}$.

Figura 7. PERFIL DE INTENSIDAdes PARA RCO $=4,8 \mu \mathrm{m}$ y $\lambda=1300 \mathrm{~nm}$ EN SIMULACIÓN

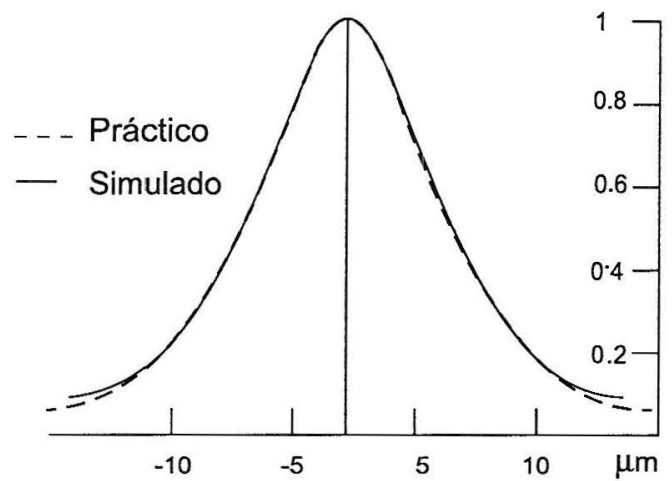

FIGURA 8. PERFIL DE IR PARA RCO $=4,8 \mu \mathrm{m}$ y $\lambda=1300 \mathrm{~nm}$ EN SIMULACIÓN

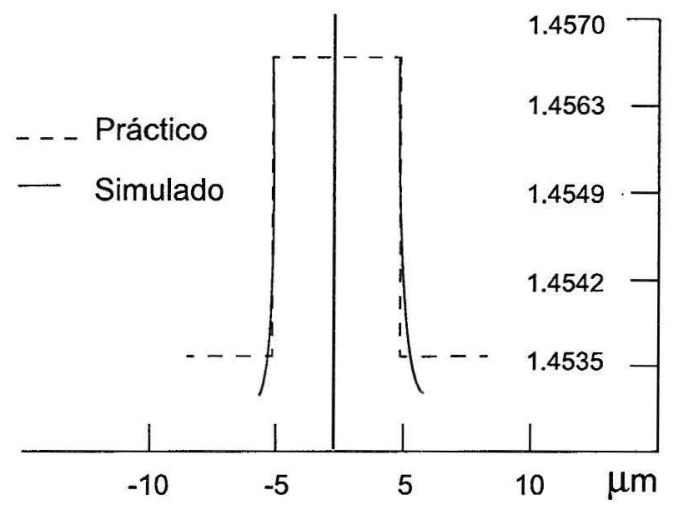

Los perfiles simulados de intensidad y de refracción, mostrados en las figuras 7 y 8 , luego del proceso interactivo de variación del perfil de índice son lo mejor ajustados a los valores experimentales. La función de mérito arrojó un valor mínimo de $0.8 \%$. 
Los resultados muestran que la técnica es muy acertada para determinar el perfil de refracción de una fibra óptica.

Algunos factores que deben tenerse en cuenta en la presente simulación y que inciden en los resultados:

- La discretización realizada al perfil de índice de la fibra experimental no es la misma que el software realiza e implementa a la fibra simulada, esto hace que los campos eléctricos comparados en la función de mérito no correspondan a la misma posición radial.

- El proceso de discretización utilizado es más denso al centro geométrico de la fibra y disminuye a medida que se aleja axialmente, éste proceso asigna vectores de campo eléctrico a áreas cada vez mayores a medida que la densidad de elementos finitos disminuye.

- El perfil de índice de refracción experimental, como es de esperarse de los procesos de fabricación, no es el perfil de paso que idealmente se construye con la ley de perfil de potencia [1]. Esto hace que haya en la reconstrucción utilizada una dificultad para resolver con exactitud.

- El proceso algorítmico no logró ser automatizado: el ingreso de nuevos parámetros al inicio de la reconstrucción exige que se digiten nuevamente los valores de radios, índices, arcos, porciones angulares y demás, cada vez que se ingresa al bucle.

\section{CONCLUSIONES Y RECOMENDACIONES}

El análisis de literatura actual y referente a la reconstrucción de perfiles de índice muestra la necesidad de conocer el PIR y, sin embargo, éstos son todavía susceptibles de mejorar según la opinión de académicos con interés en el área de fibras ópticas y la experiencia venida en este trabajo.

Los resultados del presente trabajo, muestran que la técnica da buenos resultados en la reconstrucción del perfil. Por la versatilidad de ella, se espera pueda ser ampliada y mejorada para aplicaciones 
en fibras ópticas especiales y en guías de onda dieléctricos. La posibilidad de ampliar este modelo a otras fibras ópticas con otros perfiles de refracción para que permitan en próximos desarrollos cubrir la amplia gama de perfiles de índice utilizados hoy en las fibras ópticas.

$\mathrm{El}$ algoritmo puede ser mejorado en los siguientes aspectos:

- Las subrutinas que fueron embebidas pueden ser modificadas para que consulten los datos de inicialización desde un archivo evitando que sean ingresados por teclado.

- Utilizar igual discretización en los perfiles de índice experimental y simulado, esto es, el mismo diferencial de radio dr, para ambos casos y así disminuir el error en la función de mérito.

- Considerar la posibilidad de variar de manera reiterativa sobre la ley de potencia del perfil para que se ajuste al modelo real y no fijarlo para todo el ciclo de reconstrucción.

- Distribuir de manera diferente la densidad de elementos finitos en el borde del núcleo y en revestimiento, ello contribuirá a tener mejor valoración en los sectores circulares alejados del centro geométrico de la fibra.

\section{Bibluografía}

ANTONAKOS, James L., MANSFIELD Jr, Kenneth C., Programación estructurada en C. Prentice Hall Madrid, 1997, 584p.

BIBRA, Mark L. Von and ROBERTS, Ann, Refractive Index Reconstruction of Graded Buried Channel Waveguides from their Mode Intensities, Revista: Journal of Lightwave Technology. vol. 15, $\mathrm{n}^{\circ} 9$ September 1997, pp 1695-1699.

CACCAVALE, F., A finite differences method for the reconstruction of refractive index profile from near-field measurements. Revista: Journal of Lightwave Technology. vol. 16, n 7 July 1998, pp 1348 - 1352.

DEITEL, H. M., DEITEL, P. J., C., How to Program, Prentice Hall New Jersey, 588p. 
DRAGOMAN, D., MEUNIER, J. P., Recovery of longitudinally variant refractive index profile from the measurement of the Wigner transform. Revista: Journal Optics Communications. vol. 153, 1998, pp 360 - 367.

GISIN, N.; PASSY, R. and PERNY B. Optical Fiber Characterization by Simultaneous Measurement of the Transmitted and Refracted Near Field. Revista: Journal of Lightwave Technology. vol. 11, $\mathrm{n}^{\circ} 11$ November 1993, p 1875.

GLOGE, D., MARCATILI, E. A. Multimode theory of graded-core fibers. Revista: Journal Bell. Syst. Tech. vol. 52, n9 1973, p 1563 -1578.

HECTH, Eugene Óptica, Addison Wesley Iberoamericana S.A. Madrid, 2000, 722p.

MANSOUR, I., CACCAVALE, F., An Improved Procedure to Calculate the Refractive Index Profile from the Measured Near-Field Intensity. Revista: Journal of Lightwave Technology. vol. 14, n³ March 1996, p 432.

MENESES S., Ruth del S., Análisis del perfil de indice de refracción de una fibra óptica, para comuncaciones, desde el método de elementos finitos y la técnica del campo cercano. Trabajo de Grado Universidad Nacional de Colombia, Sede Medellín, 2000.

MORISHITA, K. Index Profiling of Three-Dimensional Optical Waveguides by the Propagation-Mode Near-Field Method. Revista: Journal of Lightwave Technology. vol. LT-4, $\mathrm{n}^{\circ} 8$ August 1986, p 1120.

TORRES, Pedro, GUZMAN, A., Amplificador de fibra óptica dopada con Erbio. Memorias Cuarto Encuentro Nacional de Óptica Universidad Nacional de Colombia Junio 29-Julio 1 de 1994.

Complex finite element method applied to the análysis of optical waveguide amplifiers. Revista: Journal of Lightwave Technology. vol. $15, \mathrm{n}^{\circ} 3$ 1997, p546.

TOWSEND, P. D. Optical Effects of Ion Implantation. Revista: Rep. Prog. Phys., vol. 50, 1987, pp 501-558.

ZIENKIEWICZ, O. C., MORGAN, K., Finite Elements And Approximation, A Wiley-Intersciencie Publication, New York, 1983, 328p. 